that tyrosinase expression has independent prognostic value in patients with advanced metastatic melanoma.

Original article Quaglino P et al. (2007) Prognostic relevance of baseline and sequential peripheral blood tyrosinase expression in 200 consecutive advanced metastatic melanoma patients. Melanoma Res 17: 75-82

\section{Collagen XXIII-a promising biomarker for prostate cancer metastasis}

Banyard and co-workers have previously reported a novel collagen, type XXIII, which is highly expressed in metastatic rat prostate cancer cells compared with poorly metastatic cell lines. Now, these researchers demonstrate that collagen XXIII has prognostic value in human prostate cancer.

Immunohistochemical staining for collagen XXIII was conducted on samples obtained from patients with prostate cancer who had undergone radical prostatectomy at the University Hospital Ulm, Germany, during the period 1986-2002. Only very low levels of collagen XXIII staining were detected in benign prostate epithelia. Collagen XXIII expression was considerably higher in primary prostate tumor samples than in benign samples, but the highest level of collagen XXIII expression was found in those with distant metastases. A retrospective study of 76 patients demonstrated that a high level of collagen XXIII staining was significantly associated with time to prostate-specific antigen (PSA) failure; patients with high levels of collagen XXIII had a median time to PSA failure of 8.1 months, compared with 5.0 years for those with low levels of this biomarker. Multivariate analysis revealed collagen XXIII to have prognostic value for PSAspecific disease recurrence, independent of tumor stage, Gleason score and preoperative PSA level.

On the basis of these results, and the finding that collagen XXIII can be detected in urine samples of patients with prostate cancer, the authors conclude that this protein might prove to be a useful noninvasive biomarker for prostate cancer recurrence.

Original article Banyard J et al. (2007) Collagen XXIII expression is associated with prostate cancer recurrence and distant metastases. Clin Cancer Res 13: 2634-2642

\section{Increased risk of cancer in hyperthyroid patients treated with radioactive iodine}

Radioactive iodine (RAI) is the first-line treatment for hyperthyroidism; however, there are serious concerns about the subsequent risk of cancer in patients who receive this therapy. A recent study by Metso et al. has evaluated the risk of cancer following RAI treatment.

This study included 2,793 Finnish patients with hyperthyroidism (457 men and 2,336 women) treated with RAI between January 1965 and June 2002 (group A) and 2,793 reference subjects matched for age and sex (group B). The median age for both groups was 62 years (quartile range, $50-75$ years) and the median follow-up times were 9.8 and 10.0 years for groups A and B, respectively. The overall cancer incidence was greater in group $A$ than in group $B$ (rate ratio $[R R] 1.25$ ). Patients treated with RAl had increased incidences of breast (RR 1.53), stomach (RR 1.75) and kidney (RR 2.32) cancers, and of cancer at an unspecified site (RR 2.22) when compared with the control group (number needed to harm 418). Patients treated with high doses of RAl (370-2,664 MBq) had an increased cancer risk (RR 1.47) when compared with group $\mathrm{B}$, and the risk of cancer increased with increasing cumulative doses of RAl. Although the incidence of cancer was higher in group A than in group B for all age groups, statistically significant increases in cancer incidence were observed in patients who were 5059 years or $70-98$ years of age when treated (RR 1.44 and 1.39, respectively).

This study demonstrates that the risk of cancer, especially breast, stomach and renal cancer, is increased in patients treated with RAI.

Original article Metso S et al. (2007) Increased cancer incidence after radioiodine treatment for hyperthyroidism. Cancer 109: 1972-1979

\section{Widespread immunization of young women could reduce the burden of HPV-related disease}

Cervical cancer is one of the leading causes of cancer-related death in women. Almost all cervical cancers are caused by human papillomaviruses (HPVs), with HPV-16 and HPV-18 responsible for approximately $70 \%$ of 